Original Article

\title{
Taurolidine: A New Alternative Agent for the Management of the Postoperative Air Leak
}

\author{
Chun Sung Byun, MD, ${ }^{1}$ In Kyu Park, MD, PhD, ${ }^{1,2}$ Hyo Sup Shim, MD, PhD, ${ }^{3}$ \\ Mi Kyung Bae, MD, ${ }^{1}$ Chang Young Lee, MD, ${ }^{1}$ and Kyung Young Chung, $\mathrm{MD}^{1}$
}

\begin{abstract}
Purpose: To evaluate the efficacy of taurolidine instillation on postoperative air leak by clinical study and to investigate the mechanism of action by animal experiments.

Methods: Outcomes of taurolidine instillation in patients with postoperative air leak were retrospectively reviewed. $100 \mathrm{ml}$ of $1 \%$ taurolidine solution was instilled through a chest tube in patients with postoperative air leak lasting 4days or more. Success was defined as cessation of air leak within 24 hours with full expansion of lung on chest radiograph and subsequent removal of chest tube within another 24 hours. The effect of taurolidine instillation was estimated by development of adhesion and thickening of the visceral pleura in rabbits. The study group was compared with a control group and a talc poudrage group.

Results: Out of 75 patients, the success rate was $66.7 \%$. Out of 96 total attempts, taurolidine instillation was successful in 55\%. In animal experiments, pleural adhesion was minimal in the taurolidine and control groups, whereas the talc group showed more adhesions. The taurolidine group showed a moderate visceral pleural thickening. The talc group showed severe visceral pleural thickening, whereas the control group showed mild thickening.

Conclusion: Taurolidine is an effective alternative agent for the management of air leak. A chemical sclerosing effect is suggested as the mechanism of air leak cessation
\end{abstract}

Keywords: taurolidine, instillation, air leak

\section{Introduction}

In spite of its non-life threatening nature, air leak is one of the most important complications in thoracic surgery. Air leak prolongs chest tube indwelling time and

${ }^{1}$ Department of Thoracic and Cardiovascular Surgery, Yonsei University College of Medicine, Seoul, Korea

${ }^{2}$ Department of Thoracic and Cardiovascular Surgery, Seoul National University Hospital, Seoul, Korea

${ }^{3}$ Department of Pathology, Yonsei University College of Medicine, Seoul, Korea

Received: December 19, 2011; Accepted: February 15, 2012 Corresponding author: In Kyu Park. Department of Thoracic and Cardiovascular Surgery, Seoul National University Hospital, 101 Daehak-no, Jongro-gu, Seoul 110-744, Korea

Email: ikpark@snu.ac.kr

(C)2012 The Editorial Committee of Annals of Thoracic and Cardiovascular Surgery. All rights reserved. thus increases pain, risk of infection, and cost of care. ${ }^{1)}$ Although intraoperative prevention is the most effective method to decrease air leak, postoperative intervention is necessary in some patients. Chemical pleurodesis is an active modality for the management of air leak. There is currently no ideal regimen for chemical pleurodesis, and many agents (tetracycline, iodopovidone, autologous blood, talc, OK432, etc.) have been used for this procedure. $^{2-5)}$ Availability of these agents is not universal. Talc is the most preferred and effective agent for pleurodesis in the many countries. However, asbestos-free talc is hardly available in some country, and this situation is same in our country. So, alternative, inexpensive and widely available agents are necessary. ${ }^{3)}$ Taurolidine[bis(1,1-dioxoperhydro-1,2,4-thiadiazinyl-4)-methane] (TRD) is a non-antibiotic antimicrobial and antilipopolysaccharide agent. ${ }^{6-8)}$ TRD can be administered intravascularly or intracavitarly 
and results in no serious side effects such as hypersensitive reaction, except transient, local irritation symptoms. $^{7-9)}$ TRD irrigation has been performed to treat peritonitis or empyema. ${ }^{10)}$ Recently, the anti-adherent effect of taurolidine in cancer cells was revealed. ${ }^{11,12)}$ There have been no reports on TRD pleurodesis. We have been used TRD for pleural space irrigation in patients with empyema and observed regional chest pain and faster air leak cessation after TRD irrigation in postoperative patients. These findings suggested that TRD can be used for the management of air leak. Thus, we started to use TRD as an alternative agent for the management of postoperative air leak in the mid-1990s and observed enhanced air leak cessation in these patients. This study aimed to evaluate the pleurodesis effect of TRD through a retrospective clinical study and animal experiments. The clinical study was intended to analyze the clinical efficacy and safety of TRD pleurodesis for the treatment of air leak. The animal experiment was intended to investigate the possible mechanism of TRD pleurodesis by evaluating the changes in the pleura after TRD pleurodesis.

\section{Materials and Methods}

\section{Clinical study}

We performed a retrospective analysis of the clinical results of patients who underwent TRD instillation for the management of air leak from November 2005 to August 2010. The indication for the TRD instillation was moderate air leak persisting more than 4 days after operation or tube thoracostomy. Data, such as age, sex, diagnosis, type of procedure, instillation agents, instillation number, success or failure of instillation, subjective pain score (none, mild, moderate, or severe), and development of fever (tympanic temperature $>37.8^{\circ} \mathrm{C}$ ) were obtained by review of medical records. TRD instillation was performed at the bedside through an indwelling chest tube. The TRD instillation fluid was prepared with $50 \mathrm{ml}$ of $2 \%$ TRD solution (Taurolin ${ }^{\circledR}$, Samjinpharma, Seoul, Korea) and 50 $\mathrm{ml}$ of normal saline. ${ }^{10,13)}$ Patients were premedicated with analgesics, and $10 \mathrm{ml}$ of $1 \%$ lidocaine was instilled via chest tube before instillation. The chest tube was not clamped, and the tube line was lifted up 3 feet off the ground. Patients were rotated over 30 minutes to 1 hour through bilateral lateral decubitus, supine, sitting, and knee-chest positions. The success of instillation was defined as cessation of air leak within 24 hours after instillation with full expansion of lung on chest radiograph and subsequent removal of chest tube within another 24 hours. If the instillation was unsuccessful, instillation was repeated using TRD or other agents, such as autologous blood, OK432. The air leak necessary more than three episodes of instillation was regarded as a failure of TRD instillation. The primary end point was the success rate of TRD instillation, and the secondary end point was the pattern of adverse events related to TRD instillation. The Chi-square method was used for the comparison of success rates between subgroups. This study was approved by the Institutional Review Board of Severance Hospital, Yonsei University Health System.

\section{Animal experiment}

Nine New Zealand white rabbits weighting $3 \mathrm{~kg}$ each were divided into three groups - control, TRD, and talc. After induction of general anesthesia with endotracheal intubation and mechanical ventilation, the animals were placed in left lateral decubitus position. A 3 centimeterlong lateral thoracotomy was performed in right 6th intercostal space, and intrapleural instillation was done. In the control group, no agent was instilled into the pleural cavity. In the TRD group, TRD fluid composed of $3 \mathrm{ml}$ of 2\% TRD (Tauroline ${ }^{\circledR}$, Samjinpharma, Seoul, Korea) and $3 \mathrm{ml}$ of normal saline was instilled. In the talc group, talc poudrage was done with $0.3 \mathrm{gm}$ of talc powder (Talc ${ }^{\circledR}$, Kookjeon pharmaceuticals, Seoul, Korea). After instillation of the agents, the thoracotomy was closed. Pneumothorax was drained via a $16 \mathrm{Fr}$ rubber catheter connected to a water seal bottle by Valsalva maneuver. The drainage catheter was removed before awakening, after confirmation that there was no air leak through the catheter. Two weeks later, the status of the pleura was evaluated through thoracotomy after the animals were euthanized. Specimens of each of the three lobes of the right lung and one lobe of the left lung were obtained for microscopic examination. Adhesion was graded as none $(-)$, loose $(+)$, or dense $(++)$. The area of adhesion was classified as localized-single (+), localized-multiple $(++)$, or generalized $(++)$. The thickness of the visceral pleura was measured by an image analyzer (InnerView v2.0, Innerview Corp., Seongnam, Korea) with digital microscopic image on hematoxylin-eosin stain. The thickness of the left lung represented the thickness of normal visceral pleura in rabbits. The thickness of the thickest portion was chosen for the representative value in each subject. Animal experiment was approved by the Committee for the Care and Use of Laboratory Animals of Yonsei University College of Medicine. 
Table 1 Patient distribution by disease

\begin{tabular}{lc}
\hline Diagnosis & $75(\mathrm{n})$ \\
\hline Lung cancer & 33 \\
Metastatic lung cancer & 7 \\
Benign lung tumor & 3 \\
Primary pneumothorax & 11 \\
Secondary pneumothroax & 10 \\
Bullous lung disease & 3 \\
Bronchiectasis & 2 \\
Aspergilloma & 2 \\
Destroyed lung & 1 \\
Empyema & 3 \\
Mediastinal tumor & 3 \\
\hline
\end{tabular}

\section{Results}

\section{Clinical study}

Out of 75 patients who underwent TRD instillation, 41 patients (54.7\%) were male and mean age was $54.6 \pm 17.7$ years (19-84). Majority of patients had lung cancer (44\%) or spontaneous pneumothorax (28\%) (Table 1). Seven patients with secondary spontaneous pneumothorax had undergone tube thoracostomy and 6 patients in operation group had undergone a non-pulmonary resection operation. A total of 50 patients (66.7\%) achieved success air leakage cessation with TRD instillations only. Out of 96 total attempts, TRD instillation was successful in 55 (52.1\%). The air leak was stopped on the first attempt in 41 patients (54.7\%). A second TRD instillation was successful in 9 patients (42.9\%) out of 21 patients (Table 2). There was no statistically significant difference in success rates between the operation group and tube thoracostomy group $(55.9 \%$ vs. $42.9 \%, \mathrm{p}=0.69)$. The sublobar resection group and lobar resection group showed similar success rates $(46.2 \%$ vs. $58.3 \%, \mathrm{p}=0.44)$. The most common complication of TRD instillation was regional pain. Every patient complained of mild to moderate regional pain. However, no patients experienced severe pain. Fever developed in 6 patients but resolved within 24 hours. There were no other regional or systemic adverse events.

\section{Animal experiment}

All subjects survived until the 14th day, except one that died on the night of the 13th day. All subjects were included in the analysis. The results for each subject are described in Table 3. Adhesion was noted in two subjects, in both the TRD and talc groups. There was no adhesion in the control group. The TRD group showed only single localized adhesions, whereas the talc group showed multiple localized adhesions. No dense adhesion was
Table 2 Success rate in each round of taurolidine pelurodesis

\begin{tabular}{lcrcc}
\hline Instillation & Taurolidine & $\begin{array}{c}\text { Success } \\
(\%)\end{array}$ & Fail & $\begin{array}{c}\text { Other } \\
\text { agents }\end{array}$ \\
\hline 1st & 75 & $41(57.6 \%)$ & 34 & \\
2nd & 21 & $9(47.2 \%)$ & 12 & 13 \\
Total & 96 & $50(54.1 \%)$ & 46 & \\
\hline
\end{tabular}

Table 3 Results of animal experiment

\begin{tabular}{ccccc}
\hline subject & group & $\begin{array}{c}\text { Adhesion } \\
\text { grade }\end{array}$ & $\begin{array}{c}\text { Adhesion } \\
\text { area }\end{array}$ & $\begin{array}{c}\text { Thickness of } \\
\text { visceral pleura } \\
(\mu \mathrm{m})\end{array}$ \\
\hline 1 & control & - & - & 132.3 \\
2 & control & - & - & 113.6 \\
3 & control & - & - & 22.7 \\
4 & taurolidine & + & + & 499.7 \\
5 & taurolidine & + & + & 248.4 \\
6 & taurolidine & - & - & 72.1 \\
7 & talc & + & ++ & 940.0 \\
8 & talc & + & ++ & 468.0 \\
9 & talc & - & - & 76.8 \\
\hline
\end{tabular}

noted in any subjects. The mean thickness of normal rabbit pleura was $15.0 \pm 7.9 \mu \mathrm{m}$ (6.7-22.4). Visceral pleural thickening was observed in all groups (Fig. 1). The control group showed the mildest thickening of the visceral pleura. The TRD group showed more visceral pleural fibrosis than the control group. The degree of visceral pleural thickening of the TRD group was less severe than that of the talc group. Minimal parenchymal damage was observed in the TRD group (Fig. 2).

\section{Discussion}

We demonstrated that TRD instillation facilitates air leak cessation. The results of the animal experiment suggest that the presumptive mechanism may be direct chemical irritation and sclerosing effects resulting in thickening of the pleura rather than inflammation resulting in pleural symphysis. Various alternative sclerosing agents are used for the management of air leak when popular agent, such as talc, tetracycline are not available. $^{2-5)}$ Our experience for the faster air leak cessation after TRD irrigation in patients with postoperative air leak and empyema lead us to use TRD as an alternative sclerosing agent for the management of air leak. TRD, a derivative of taurine, is a non-antibiotic antimicrobial agent with broad antibacterial and antifungal properties. ${ }^{7,10,13)}$ TRD has also been reported to 


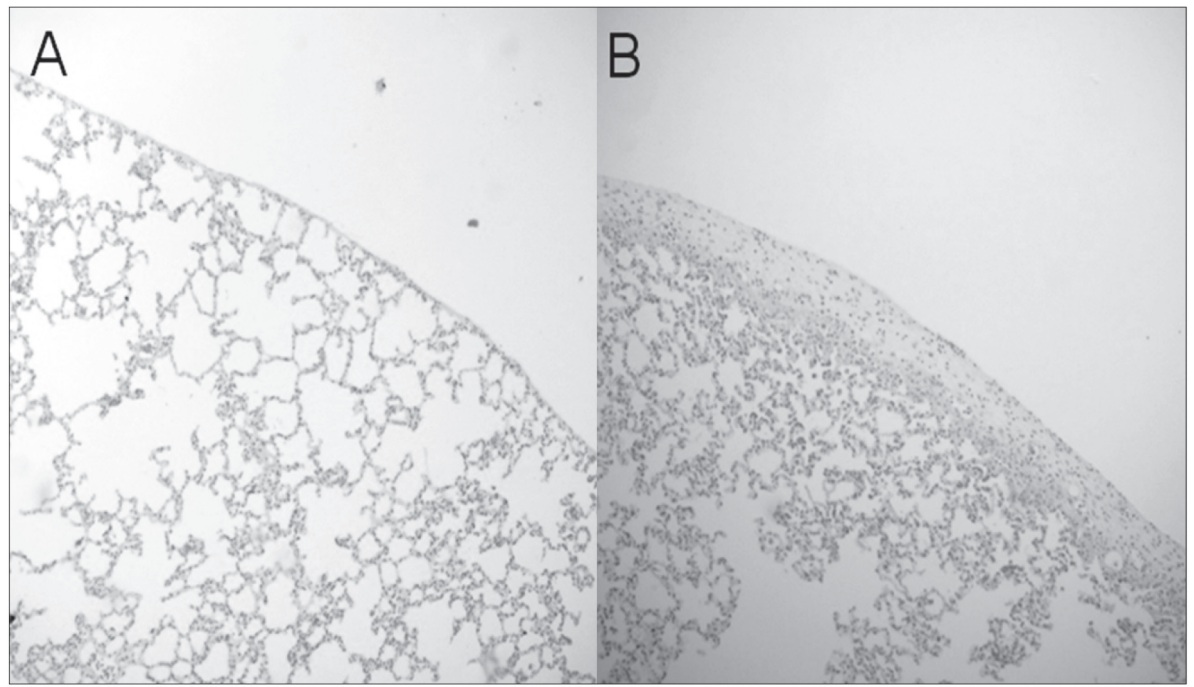

Fig. 1. Microscopic image of visceral pleura.

A: Normal untreated visceral pleura of rabbit $(\mathrm{HE}, \times 100)$, B: Thickend visceral pleura in taurolidine group $(\mathrm{HE}, \times 100)$, The microscopic findings show pleural fibrosis. There is no active inflammation and parenchymal damage.

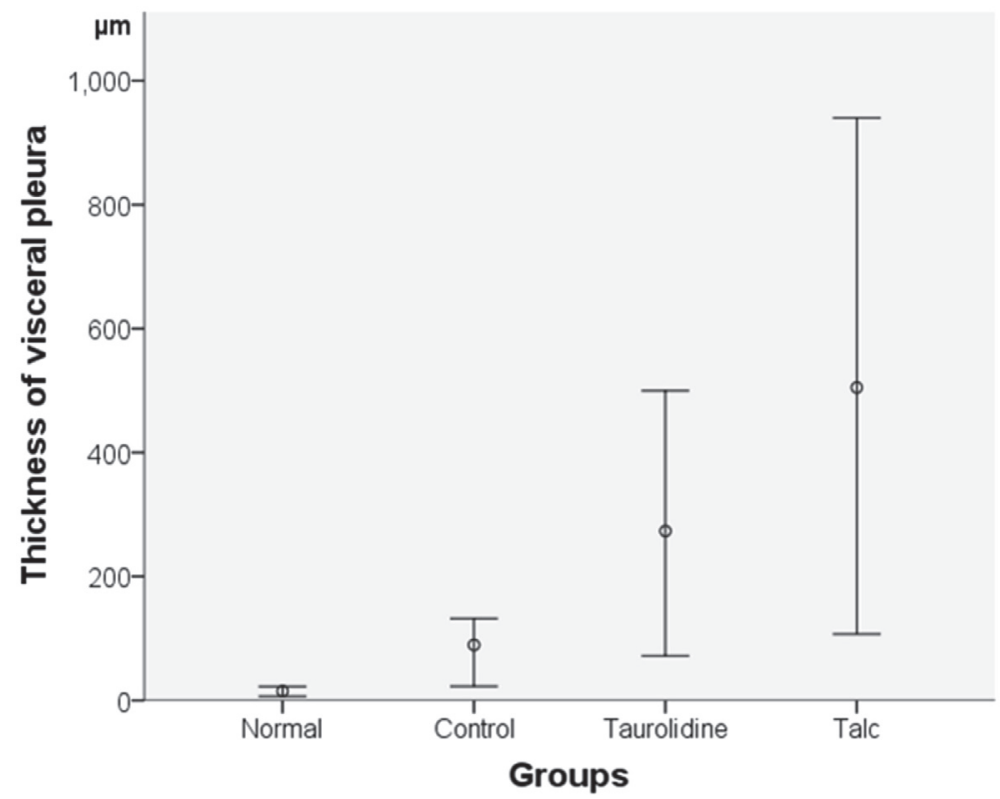

Fig. 2 Thickness of visceral pleura in each group.

have anti-adherent, anti-inflammatory, and antitumor effects. Gorman et al. and Blenkharn et al. observed that TRD inhibits bacteria from adhering to human epithelial tissue and biomaterials in vitro. ${ }^{14,15)}$ Tarhan et al. reported that TRD inhibits peritoneal adhesion most probably by altering peritoneal fibrinolytic activity. ${ }^{16)}$ TRD reduces inflammation through the inhibition of proinflammatory cytokines such as tumor necrosis factor alpha (TNF- $\alpha$ ) and interleukin-1. ${ }^{17)}$ The antineoplastic effect of TRD was reported to result from an anti-angiogenetic effect and the induction of apoptosis. ${ }^{11,18)}$ The clinical application of TRD is an antiseptic. It has been used as an intraperitoneal antiseptic for the treatment and prevention of infection in contaminated surgery. Intrapleural instillation of TRD has also been performed for the treatment of empyema thoracis. ${ }^{10)}$ Recently, a commercial venous catheter lock solution containing TRD was introduced to prevent catheter related infection. ${ }^{19)}$ We initially used TRD as an 
antiseptic for empyema, and then began to use TRD for instillation after recognizing its effect on early air leak cessation. The success rate of a first TRD instillation in this study was $54.7 \%$. This clinical result is compatible with the results of other agents. The reported success rates of tetracycline, iodopovidone, and autologous blood are range from $32 \%-74 \%{ }^{2,3,5)}$ Pleural symphysis, induced by pleural inflammation, fibrosis, neovascularization, and collagen deposition, is regarded as the most important effect of chemical pleurodesis required to achieve good pleurodesis. ${ }^{20,21)}$ However, the established features of TRD are contradictory to the requirements of the ideal pleurodesis agent. Peritoneal adhesion preventing property of TRD is contradictory to the requirement of ideal pleurodesis agent. ${ }^{16,22)}$ We hypothesized that a chemical irritation effect might be responsible for air leak cessation in TRD instillation, because the procedure induced regional irritation symptoms (chest pain) in most patients and some degree of systemic symptoms (mild fever). In animal experiments, visceral pleural thickening was more prominent in the TRD group than in the control group. However, pleural symphysis was mild and occasional. These findings indicate that TRD results in immediate air leak cessation by direct sclerosis of the pleural surface, and not by inflammation and subsequent pleural symphysis. So, the term "pleurodesis" which means pleural symphysis, is inappropriate and "instillation" would be a more exact term for TRD. The anti-adhesion effect of TRD is a drawback for TRD instillation in spontaneous pneumothorax or malignant effusion, in which pleural symphysis is important to prevent recurrence. However, this same anti-adhesion effect is beneficial in the management of postoperative air leak, which needs not permanent pleural symphysis but immediate air leak cessation, because pleural symphysis can decrease pulmonary function. ${ }^{23,24)}$ TRD is known as a safe drug without severe toxicity. Its most common reported side effect is local reaction, and systemic clinically relevant adverse events were minimal. ${ }^{819)}$ Intrapleural instillation of TRD also resulted in no serious adverse event. The most common complication of TRD instillation was mild to moderate chest pain lasting a few hours due to local irritation. Only 6 patients had clinically relevant fever, which resolved within 24 hours. There was no respiratory difficulty, trapped lung, local infection, obstruction of chest tube, or allergic reaction. TRD has several additional advantages. The preparation of TRD solution for bedside instillation is very easy because it is already a liquid preparation, unlike talc powder. The antimicrobial prop- erties of TRD can prevent infection related to prolonged chest tube indwelling or instillation procedure, and furthermore can treat superimposed infection. TRD is readily available in many countries around the world. Finally, TRD is a cheap agent, costing only 5 US dollars for a single episode of instillation. There are limitations in the current study. First, clinical analysis was done retrospectively with a single cohort without comparison groups. Therefore, a randomized controlled trial is necessary to validate the efficacy of TRD. Additionally, animal experiments were done on normal pleura. Subsequent experiments on injured visceral pleura are necessary.

\section{Conclusion}

Intrapleural instillation of TRD is a safe and effective alternative method for the management of postoperative air leak. The presumptive mechanism of air leak cessation is a direct chemical sclerosis.

\section{Acknowledgements}

This study was supported by a faculty research grant of Yonsei University College of Medicine (6-2008-0171).

\section{Disclosure Statement}

No disclosure.

\section{References}

1) Liberman M, Muzikansky A, Wright $C D$, et al. Incidence and risk factors of persistent air leak after major pulmonary resection and use of chemical pleurodesis. Ann Thorac Surg 2010; 89: 891-7.

2) Agarwal R, Aggarwal AN, Gupta D. Efficacy and safety of iodopovidone pleurodesis through tube thoracostomy. Respirology 2006; 11: 105-8.

3) Dikensoy O, Light RW. Alternative widely available, inexpensive agents for pleurodesis. Curr Opin Pulm Med 2005; 11: 340-4.

4) Miller Q, Meschter C, Neumaster T, et al. Comparison of pleurodesis by erythromycin, talc, doxycycline, and diazepam in a rabbit model. J Surg Educ 2007; 64: 41-5.

5) Rinaldi S, Felton T, Bentley A. Blood pleurodesis for the medical management of pneumothorax. Thorax 2009; 64: 258-60.

6) Watson RW, Redmond HP, Mc Carthy, et al. Taurolidine, an antilipopolysaccharide agent, has immunoregulatory properties that are mediated by the amino acid taurine. J Leukoc Biol 1995; 58: 299-306. 
7) Browne MK, MacKenzie M, Doyle PJ. C controlled trial of taurolin in established bacterial peritonitis. Surg Gynecol Obstet 1978; 146: 721-4.

8) Gong L, Greenberg HE, Perhach JL, et al. The pharmacokinetics of taurolidine metabolites in healthy volunteers. J Clin Pharmacol 2007; 47: 697-703.

9) Koldehoff M, Zakrzewski JL. Taurolidine is effective in the treatment of central venous catheter-related bloodstream infections in cancer patients. Int J Antimicrob Agents 2004; 24: 491-5.

10) Conlan AA, Abramor E, Delikaris $P$, et al. Taurolidine instillation as therapy for empyema thoracis. A prospective study of 50 patients. S Afr Med J 1983; 64: 653-5.

11) Jacobi $C A$, Menenakos $C$, Braumann C. Taurolidine--a new drug with anti-tumor and anti-angiogenic effects. Anticancer Drugs 2005; 16: 917-21.

12) McCourt M, Wang JH, Sookhai S, et al. Taurolidine inhibits tumor cell growth in vitro and in vivo. Ann Surg Oncol 2000; 7: 685-91.

13) Gallieni M, Chiarelli G, Olivi L, et al. Unsuccessful application of taurolidine in the treatment of fungal peritonitis in peritoneal dialysis. Clin Nephrol 2011; 75: 70-3.

14) Gorman SP, McCafferty DF, Woolfson AD, et al. Reduced adherence of micro-organisms to human mucosal epithelial cells following treatment with Taurolin, a novel antimicrobial agent. J Appl Bacteriol 1987; 62: 315-20.

15) Blenkharn JI. In-vitro antibacterial activity of noxythiolin and taurolidine. J Pharm Pharmacol 1990; 42: 589-90.
16) Tarhan OR, Barut I, Sezik M. An evaluation of normal saline and taurolidine on intra-abdominal adhesion formation and peritoneal fibrinolysis. J Surg Res 2008; 144: 151-7.

17) Marcinkiewicz J, Gluszko P, Kontny E, et al. Is Taurolidine a candidate for treatment of rheumatoid arthritis? Clin Exp Rheumatol 2007; 25: 211-8.

18) Opitz I, Sigrist B, Hillinger S, et al.Taurolidine and povidone-iodine induce different types of cell death in malignant pleural mesothelioma. Lung Cancer 2007; 56: $327-36$.

19) Bradshaw JH, Puntis JW. Taurolidine and catheter-related bloodstream infection: a systematic review of the literature. J Pediatr Gastroenterol Nutr 2008; 47: 179-86.

20) Bouros D, Froudarakis M, Siafakas NM. Pleurodesis: everything flows. Chest 2000; 118: 577-9.

21) Mitchem RE, Herndon BL, Fiorella RM, et al. Pleurodesis by autologous blood, doxycycline, and talc in a rabbit model. Ann Thorac Surg 1999; 67: 917-21.

22) Bahadir I, Oncel M, Kement M, et al. Intra-abdominal use of taurolidine or heparin as alternative products to an antiadhesive barrier (Seprafilm) in adhesion prevention: an experimental study on mice. Dis Colon Rectum 2007; 50: 2209-14.

23) Chen JS, Cheng WC, Lien HC, et al. Patterns, effects, and thoracic volume changes of thoracoscopic pleurodesis in rabbits. J Surg Res 2008; 147: 34-40.

24) Györik S, Erni S, Studler U, et al. Long-term follow-up of thoracoscopic talc pleurodesis for primary spontaneous pneumothorax. Eur Respir J 2007; 29: 757-60. 